\title{
Photoinduced Topological Insulator to Dirac Semimetal Transition in ZrTe5
}

\author{
Tatiana Konstantinova ${ }^{1}$, Lijun $\mathrm{Wu}^{2}$, Weiguo $\mathrm{Yin}^{2}$, Jing $\mathrm{Tao}^{2}$, Genda $\mathrm{Gu}^{2}$, Igor Zaliznyak ${ }^{2}$ and Yimei $\mathrm{Zhu}^{3}$
}

${ }^{1}$ Brookhaven National Lab, United States, ${ }^{2}$ Brookhaven National Laboratory, United States, ${ }^{3}$ Department of Condensed Matter Physics and Materials Science, Brookhaven National Laboratory, Upton, NY, USA, United States

Pump-probe based ultrafast electron microscopy has brought the research of condensed matter physics and materials science to a new level. Due to their different temporal response electron, spin and lattice interactions and their degrees of freedom are best investigated in time domain $[1,2,3]$. Studies of the topological aspects of electronic band structure and the emergent phases governed by topological and chiral protection reveal unusual electronic quasiparticles of Weyl, Dirac, and other exotic types with novel properties valuable for technological applications. Of particular interest is the engineering of new phases of matter using ultrafast external stimuli, such as photonic and electronic excitations, to observe, manipulate and control materials' functionality.

In this presentation, we show how a transient 3D Dirac semimetal state can be induced by a femtosecond laser pulse in a topological insulator $\mathrm{ZrTe}_{5}$ using MeV ultrafast electron diffraction (UED) [3]. We observe remarkable changes in Bragg diffraction, which are characteristic of bond distortions in the photoinduced state (Fig.1). Photoinduced atomic displacements are accurately determined through quantitative analysis of the intensity changes using the time-resolved electron crystallography method we developed. Based on the atomic positions refined from the UED, we then perform density functional theory (DFT) calculations of the electronic band structure. Our results unveil that the equilibrium state of $\mathrm{ZrTe}_{5}$ is a topological insulator with a small band gap of $\sim 25 \mathrm{meV}$. However, the gap is closed in the presence of strong spin-orbit coupling (SOC) in the photoinduced transient state, where massless Dirac fermions emerge in the chiral band structure. The time scale of the relaxation dynamics to the transient Dirac semimetal state is remarkably long, $\tau \sim$ 160ps, which is two orders of magnitude longer than the conventional phonon-driven structural relaxation (Fig.2). The long relaxation is consistent with the vanishing density of states in Dirac spectrum and slow spin-repolarization of the SOC-controlled band structure accompanying the emergence of Dirac fermions. If time permits, results using laser-free pulsed electron beams to directly visualize electromagnetic wave dynamics in interdigitated comb architecture as well as the photoinduced insulator-to-metal transition and the ensuing electronic dynamics and dielectric response in a single $\mathrm{VO}_{2}$ nanowire captured by two-color near-field ultrafast electron microscopy will also be discussed [4].

(a)

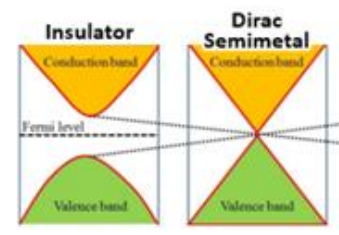

MeV-UED: Experiment $t=0$

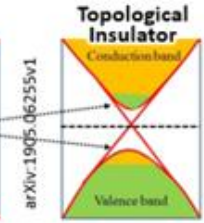

Experimental difference pattern

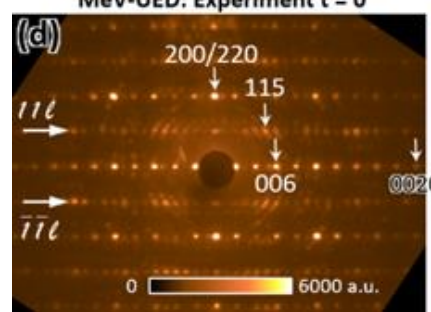

(b)

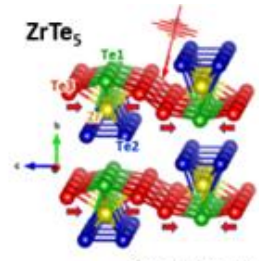

Simulated pattern: $\mathrm{t}=0$

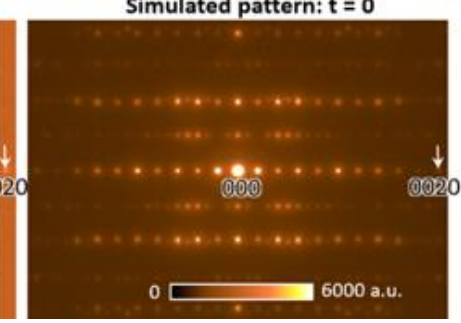

(c)

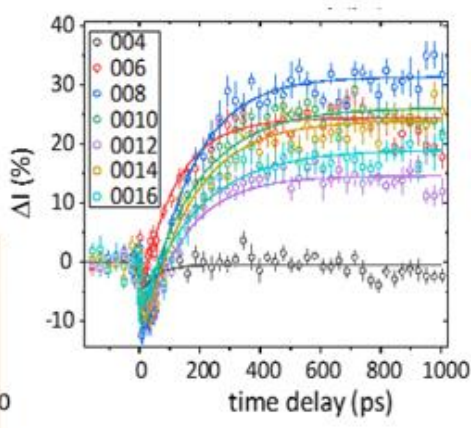

Figure 1. Fig.1 (a) Schematic of electronic band structure transitions from trivial insulator, Dirac semimetal, to topological insulator. (b) Atomic structure of ZrTe5, showing atomic displacement of Te3 upon photoexcitation.Different colors highlight the symmetrically non-equivalent apical (Te1), dimer (Te2), and zigzag (Te3) tellurium atoms forming the ZrTe3 chains and the ZrTe5 sheets. (c) Intensity change of (00L) reflections as a function of time delays. (d) Experimental UED pattern at $\mathrm{t}=0$, difference pattern and simulation. 
(a)

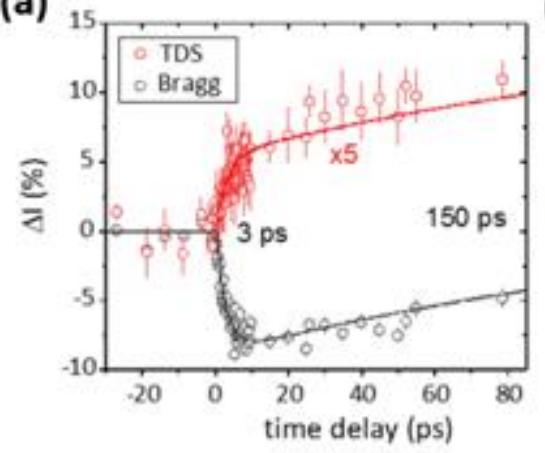

(d)

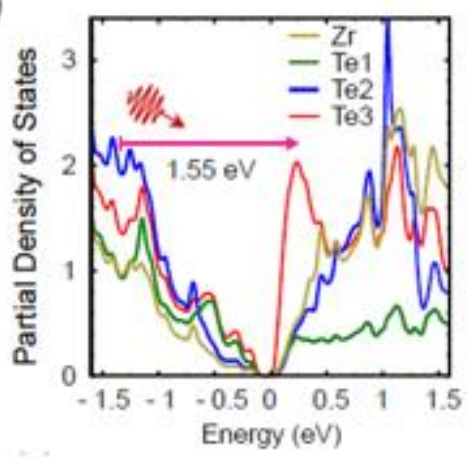

(b)

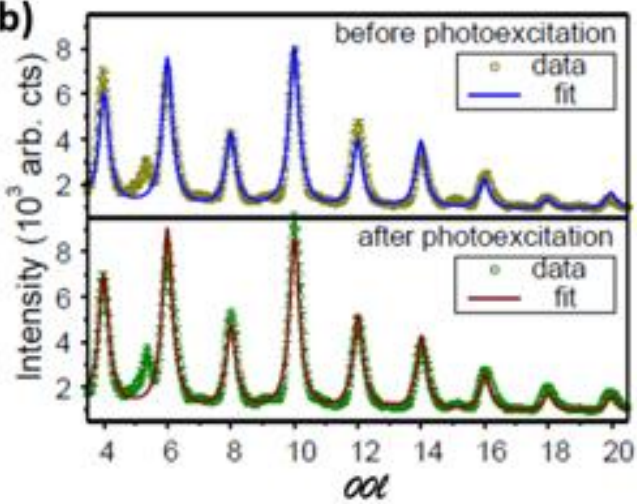

(c) Wyckoff position of Te2

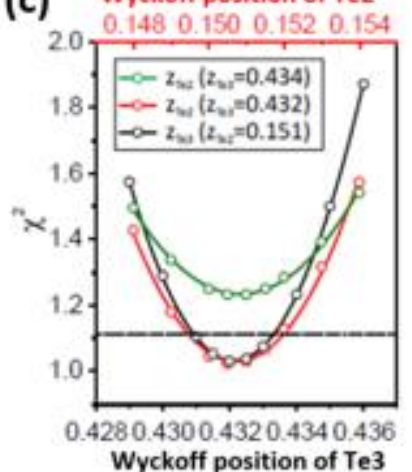

(e)

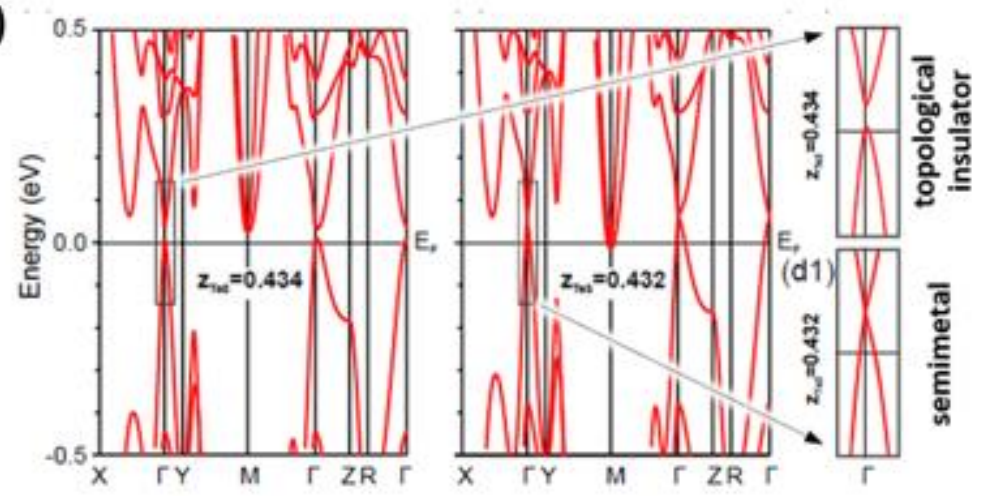

Figure 2. Fig.2 (a) Two-timescale lattice dynamics of ZrTe5, based on Bragg reflections and thermal diffuse scattering analyses. (b) Measured UED (00L) intensity profiles before and after photoexcitation. The small peak on the left side of $\left(\begin{array}{lll}0 & 0 & 6\end{array}\right)$ is a contamination from the $\left(\begin{array}{lll}1 & 1 & 4\end{array}\right)$ reflection of the small flake and was discarded in the refinement. The error bars show one standard deviation. (c) The value of $\chi 2$ quantifying the goodness of fit as a function of the Wyckoff position of Te 3 and $\mathrm{Te} 2$ along $c$ direction. (d) DOS at time zero, for Te 3 position $z T e 3=0.434$. The arrow illustrates the photoexcitation process where electrons from valence bands are excited to a Te3-dominated DOS peak near the Fermi level in the conduction band. (e) Left-panel: The weak topological insulator band structure of ZrTe5 in the equilibrium state at time zero $(z T e 3=0.434)$ showing a small direct gap of $\approx 25 \mathrm{meV}$ at the $\Gamma$ point. Mid-panel: The band structure in the photoexcited state $(z T e 3=0.432)$ has gap closed at the Dirac point, revealing a photoinduced phase transition from topological insulator to Dirac semimetal. Right-panel: Zoom in at the band structure near the $\Gamma$ point (marked by the black rectangles) in the Left- and Mid-panels, respectively.

\section{References}

[1] Fu, X.; Wang, E.; Zhao, Y.; Liu, A.; Montgomery, E.; Gokhale, V. J.; Gorman, J. J.; Jing, C.; Lau, J. W.; Zhu, Y., "Laser-free ultrafast electron microscopy of electromagnetic wave dynamics", Science Advances, 6 eabc3456 (2020).

2] Fu, X.; Barantani, F.; Gargiulo, S.; Madan, I.; Berruto, G.; Lagrange, T.; Jin, L.; Wu, J.; Vanacore, G.M.; Carbone, F.; and Zhu, Y.; "Nanoscale-femtosecond dielectric response of Mott insulators captured by two-color near-field ultrafast electron microscopy”, Nat. Comm., 11: 5770 (2020).

[3] Konstantinova, T.; Wu, L.; Yin, W.-G.; Tao, T.; Gu, G. D.; Wang, X.J.; Zaliznyak, I. A.; Zhu, Y. "Photoinduced chiral Dirac semimetal in ZrTe5", npj Quantum Materials, 5: 80 (2020)

[4] The authors would like to acknowledge J. Yang and X. J. Wang of SLAC for assistance. Work at BNL was supported by the US DOE-BES, MSED, under Contract No. DESC0012704. 